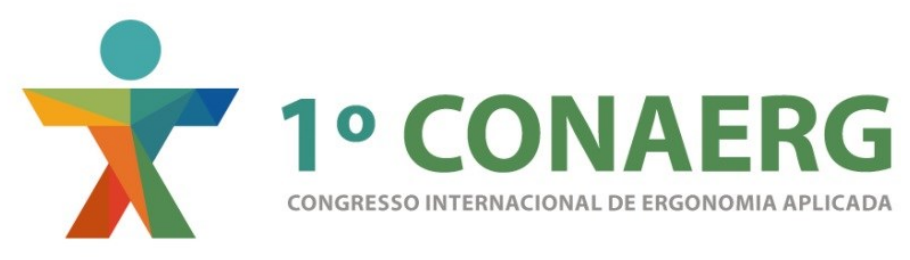

\title{
ANÁLISE ERGONOMICA DA TAREFA DOS ENFERMEIROS DAS CLÍNICAS CIRÚRGICAS DA INTERNAÇÃO DO HOSPITAL DAS CLÍNICAS DA UFPE \\ Um estudo com o uso da Termografia Digital
}

\author{
(1) José Roberto Rocha da Silva \\ (2) Márcio Marçal \\ (3) Jacqueline Augusta do Nascimento Oliveira \\ (3) Marcelo Márcio Soares
}

\begin{abstract}
RESUMO
Avaliar a atividade laboral dos enfermeiros, a partir da análise ergonômica dos postos de trabalho das clínicas cirúrgicas do hospital das clínicas da UFPE, aplicando a técnica da termografia digital. A pesquisa ocorreu por meio da captura das imagens termográficas. Os resultados evidenciam alterações fisiológicas apontadas pelos pontos de hiperradiação devido ao aumento do metabolismo cutâneo nos pesquisados, apresentadas nas áreas em destaque, sendo as regiões corporais admitidas topograficamente: as regiões da coluna cervical, da coluna torácica, lombar e dos ombros. Recomenda-se a prevenção dos transtornos musculoesqueléticos para melhorias do bem-estar no ambiente laboral.
\end{abstract}

Palavras chaves: Ergonomia. Enfermagem. Posto de trabalho. Termografia digital. 


\begin{abstract}
Assess the work activity of nurses, from the ergonomic analysis of the jobs of the surgical hospital clinics clinical UFPE, applying the technique of digital thermography. The research took place by capturing the thermographic images. The results show physiological changes indicated by points of hyper-radiation due to increased skin metabolism in the researched, presented the highlights areas, and the accepted body regions topographically: the regions of the cervical spine, thoracic spine, lower back and shoulders. It is recommended to prevent musculoskeletal disorders for welfare improvements in the work environment.
\end{abstract}

Keywords: Ergonomics. Nursing. Workstation. Digital thermography

\title{
1. INTRODUÇÃO
}

As transformações no mundo do trabalho têm refletido em mudanças na configuração do conjunto da vida individual, social e cultural. A competitividade e a produtividade se tornaram paradigmas no mundo da produção e do trabalho tornando a tecnologia e o conhecimento cientifico essenciais. Para os enfermeiros, a qualificação profissional e a qualidade dos conhecimentos produzidos, são fundamentais para o bem-estar, qualidade de vida e melhoria na área de atuação (RIBEIRO, 2003). No contexto do posto de trabalho, a tarefa do enfermeiro é permeada por múltiplas demandas de atenção provenientes da complexidade e da prestação dos cuidados, do ambiente de trabalho e da própria instituição de saúde. Essas demandas são situações que exigem do enfermeiro competências técnicas e atitudinais para conduzir a assistência de forma ágil, segura e precisa (SANTOS, 2007). Frequentemente, esses profissionais intervêm em setores particulares da economia ou em domínios de aplicação específicos. Esses últimos caracterizam-se por sua constante mudança, com a criação de novos domínios de aplicação ou do aperfeiçoamento de outros mais antigos (ABERGO, 2016). Segundo Inoue (2010), o posto de trabalho dos enfermeiros nos hospitais é caracterizado por tarefas complexas, que exige alta competência tecnocientífica para a tomada de decisões imediatas e condutas seguras ao restabelecimento do usuário. Nesse cenário, é importante prover e manter o dimensionamento adequado e qualificado de trabalhadores em enfermagem, com o objetivo de evitar sobrecarga de trabalho e, em consequência, insatisfação dos profissionais, problemas pessoais, nas relações com a equipe de saúde da unidade, adoecimentos e até afastamentos do trabalho. A Termografia Infravermelha Computadorizada utilizada na captura das imagens nos enfermeiros, mostrou sensibilidade na identificação das regiões da queixa de dor relatadas pelos profissionais desta pesquisa. As regiões corporais segmentadas para o estudo, apoiam a existência de uma disfunção funcional registradas nas alterações da temperatura na pele. É um procedimento diagnóstico que mensura o calor irradiado emitida pelo corpo. Estas medidas se expressam na forma de imagens que constituem a base para em conjunto com o exame clinico serem utilizadas auxiliando no diagnóstico de condições neuromusculares dolorosas como discopatias, síndrome de dor miofascial, miosites, lesões musculo ligamentosas, radiculopatias, distrofia simpático-reflexa, síndromes do túnel do carpo e do tarso, lesões esportivas, síndromes compressivas neurovasculares do desfiladeiro torácico e inflamações de artrites, tendinites e bursites 
(AHN, 2002). Diante disso, objetivou-se avaliar a atividade laboral dos enfermeiros, a partir da análise ergonômica dos postos de trabalho das clínicas cirúrgicas da internação do hospital das clínicas da UFPE, usando a técnica da termografia digital.

\section{MATERIAL E MÉTODO}

A escolha do tema adveio da necessidade de estudar como a atividade laboral dos enfermeiros pode comportar-se de maneira determinante, assim como os fatores pessoais e de trabalho relacionados com a ocorrência de dores nas costas entre os enfermeiros. Em particular, as lesões do sistema musculoesquelético que despertaram a atenção deste pesquisador e enfermeiro da unidade, visto constitui uma das mais importantes causas de morbidade e de incapacidade de adultos, e também pelos custos econômicos que acarretam. A pesquisa foi de natureza descritiva, exploratória com abordagem quantitativa sobre à análise ergonômica da atividade dos enfermeiros das clínicas cirúrgicas da internação do Hospital das Clínicas da UFPE. Foi realizada no Hospital das Clínicas da Universidade Federal de Pernambuco, na Ala de clínica cirúrgica, Bloco B da internação do hospital. O Hospital das Clínicas está localizado as margens da BR 101, no bairro da Cidade Universitária, em Recife. Sua localização é de fácil identificação, porém, contrapartida, o seu hall de acesso não apresenta qualquer sinalização que oriente a circulação interna de visitantes. Para coleta de dados foi aplicada a entrevista, aplicação do questionário que compreende os seguintes aspectos: (aspectos individuais, capacidade para o trabalho, questionário nórdico padrão (PINHEIRO, TRÓCOLI, \& CARVALHO, 2002), aspectos psicossociais, aspectos institucionais, aspectos pessoais, questionário escala de estresse no trabalho (PASCHOAL \& TAMAYO, 2004) e Instrumento de Escala de Estresse no Trabalho (STACCIARINI \& TRÓCOLLI, 2000), com duração de aproximadamente 20 minutos, realizada no próprio local de trabalho, relativo à percepção de riscos ergonômicos entre os enfermeiros das clínicas cirúrgicas do HC/UFPE. O Hospital das clínicas oferece consultas acerca de 33 especialidades. Por mês são atendidos, em média, 12 mil pacientes. Os recursos humanos do Hospital das Clínicas, estão distribuídos da seguinte maneira: Funcionários da UFPE - 1.534, docentes - 220, estudantes de graduação - 2.200, estudantes de mestrado/doutorado - 510, residentes - 242, estagiários curriculares -938 e voluntários -45 . Dentro deste contexto, a população tomada para a realização da pesquisa, foi composta pelos enfermeiros lotados nas clínicas cirúrgicas da internação do Hospital das clínicas no período de janeiro e fevereiro de 2016. A identificação da amostra levou em consideração os seguintes critérios: Ser enfermeiro da clínica cirúrgica; Estar em Atividade no período da pesquisa; Disponibilidade para participar da pesquisa; 6 meses de experiência de trabalho na instituição. A amostra foi composta por um total de 40 enfermeiros. A pesquisa se iniciou única e exclusivamente após a sua aprovação pelo Comitê de Ética em Pesquisa parecer número 1.349.166 e foi garantido o anonimato de todos os participantes da pesquisa. Utilizamos as seguintes etapas: a apreciação ergonômica, que é a fase de levantamento exploratório que compreende o mapeamento dos problemas ergonômicos, a diagnose ergonômica que é a fase que permite a priorização dos problemas encontrados e testagem de predições conforme (MORAES \& MONT'ALVÃO, 2010). Partindo deste princípio, utilizando a abordagem sistêmica e sistemática (sistêmica, por ser holística e sistemática, por compreender uma série de etapas e fases) do sistema alvo e do seu ambiente, é possível realizar uma intervenção ergonomizadora (MORAES \& MONT'ALVÃO, 2010). A intervenção ergonômica, em sua primeira etapa - a apreciação ergonômica - implica o enfoque sistêmico do sistema homem-tarefa-máquina. Através de 
observações exploratórias e entrevistas focalizadas, fazem-se a problematização e a sistematização do sistema homem-tarefa-máquina, que tem por objetivo a clara definição do problema a ser analisado, a partir de uma negociação com os diversos atores envolvidos aceda (MORAES \& MONT'ALVÃO, 2010). A diagnose ergonômica permite aprofundar os problemas priorizados e testar as predições (MORAES \& MONT'ALVÃO, 2010). Nesta fase de diagnose avaliaremos o desconforto corporal através das ferramentas ergonômicas: Questionário Nórdico Padronizado para Análise dos Sintomas Musculoesqueléticos, o REBA (Avaliação Rápida do Corpo Inteiro/ Rapid Entire Body Assessmente), a Termografia e a pulseira inteligente. Para isto, o questionário Nórdico Padronizado para Análise dos Sintomas Musculoesqueléticos que teve como objetivo apresentar a prevalência do problema musculoesquelético nos principais grupos articulares, dividido quatro partes: queixa e desconforto no último ano; se esse desconforto levou uma cessação das atividades do trabalhador; se esse desconforto levou você a procurar um profissional de saúde; queixa de dor e desconforto na última semana. Esse instrumento permitirá termos um mapa da prevalência da dor e do desconforto musculoesquelético no último ano e nas últimas semanas (PINHEIRO, TRÓCOLI, \& CARVALHO, 2002). Os problemas ergonômicos a ser avaliados neste posto de trabalho, estão relacionados às dimensões do mobiliário e do próprio espaço de trabalho, problemas referentes ao ambiente de trabalho, com relação a conforto térmico, ruídos e iluminação. A tarefa para análise será o trabalho dos enfermeiros nos postos de trabalho. Os dados foram tabulados em duplicata no programa EpiData 3.1, fabricado por JPA Haddad \& P Marques-Vidal \& Marco Moura, com checagem automática de consistência e amplitude dos valores para cada variável. A ferramenta "validar dupla digitação" foi utilizada para identificar possíveis erros de digitação, os quais foram identificados e corrigidos a partir dos valores originais nos questionários. Foram empregados os procedimentos de análise estatística descritiva como média e desvio padrão para variáveis numéricas, assim como, distribuição de frequência relativa e absoluta para as variáveis categóricas. A distribuição normal dos dados foi avaliada pelo teste de ShapiroWilk $(p \geq 0,05)$. Utilizou-se o teste de Qui-quadrado de Pearson para avaliação da heterogeneidade, comparando as proporções de variáveis discretas em escala nominal e associação bivariada. Quando frequências observadas foram inferiores a cinco, aplicou-se o Teste Exato de Fisher, ambos adotando um nível de significância de $5 \%$. Todos os dados coletados nesta pesquisa (entrevistas) ficarão guardados em (pastas de arquivo e computador pessoal), sob a responsabilidade do pesquisador, no endereço residencial informado no TCLE, pelo período de (mínimos cinco anos). Este estudo seguiu as normas delimitadas pela Resolução $n^{\circ} 466 / 2012$, do Conselho Nacional de Saúde. Esta resolução determina o uso compulsório do Termo de Consentimento Livre e Esclarecido o qual será assinado pelo pesquisado. A presente pesquisa foi submetida à apreciação pelo Comitê de Ética em Pesquisa do Centro de Ciências da Saúde da Universidade Federal de Pernambuco. A pesquisa se iniciou única e exclusivamente após a sua aprovação pelo Comitê de Ética em Pesquisa parecer número 1.349.166 e foi garantido o anonimato de todos os participantes da pesquisa.

\section{RESULTADOS}

O uso deste posto para os indivíduos classificados na categoria 2,5 para o feminino e 97,5 para o masculino, resultam em algumas irregularidades antropométricas que dificultam realização das atividades do enfermeiro. A Figura 1, mostra o enfermeiro executando a tarefa, no seu ambiente de trabalho. 
Figura 1 -Área de Serviço - Percentil 2,5

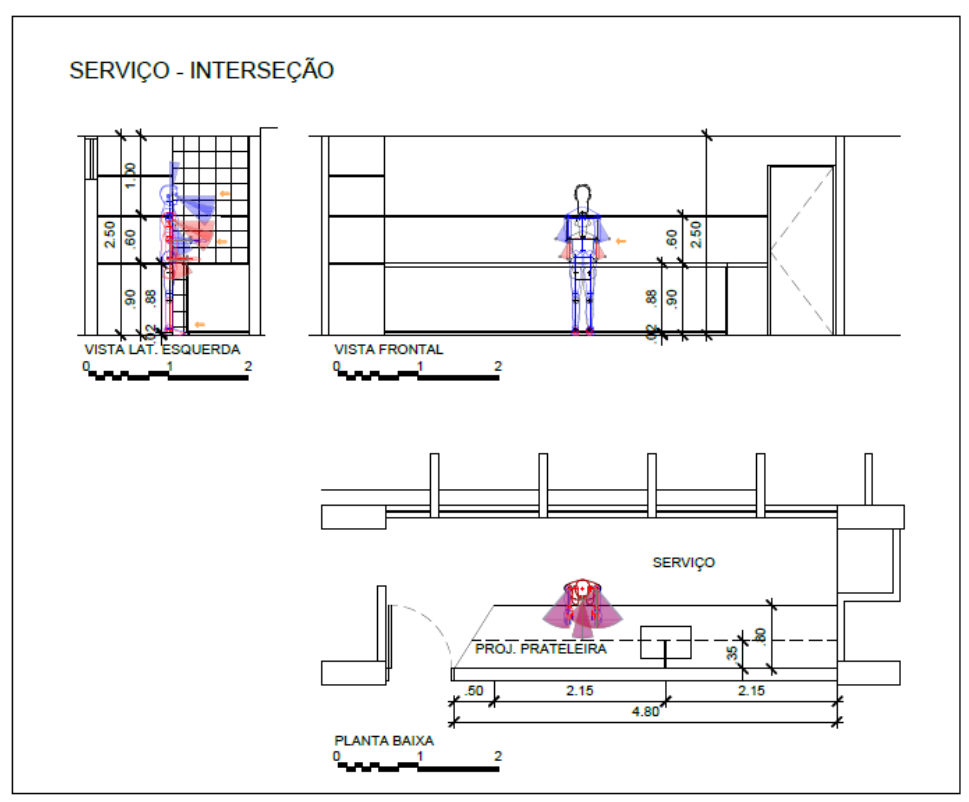

Fonte: elaboração do autor

O uso deste posto por um indivíduo classificado nesta categoria resulta em algumas irregularidades antropométricas que dificultam realização das atividades do enfermeiro. Dentre estas irregularidades foram identificadas:

a) Altura da superfície da mesa incompatível com o biótipo. Irregularidade identificada ao se avaliar o funcionário fazendo elevação do ombro acima da zona de conforto e o apoio dos membros superiores sobre a mesa com a inclinação da coluna ao se preparar as medicações. Esta condição provoca a inclinação da cabeça em um ângulo acima $15^{\circ}$, constrangendo a extensão do pescoço e também nos ombros, a prateleira acima do campo de visão, do envelope de alcance e da amplitude de movimento dos ombros, forçando o profissional a elevar a cabeça acima de $15^{\circ}$ e a esticar-se;

b) Áreas de alcance fora da região de conforto. As áreas de atividade localizadas fora do raio dos membros superiores, provocando deslocamentos com ângulos superiores as $45^{\circ}$;

c) Posição das gavetas. As gavetas são baixas exigindo a rotação e flexão excessiva do tronco e membros superiores.

A Figura 2, mostra o enfermeiro executando a tarefa do preparo das medicações, no seu ambiente de trabalho 
Figura 2 - Enfermeiro na execução da tarefa de preparo de medicação

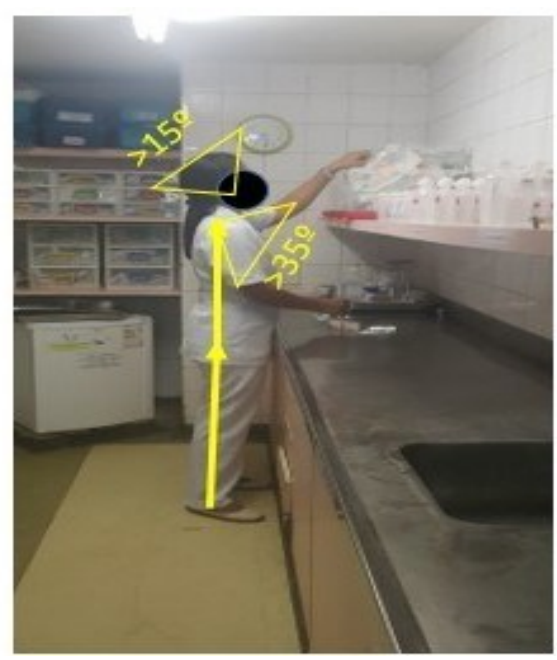

Fonte: elaboração do autor

A pontuação encontrada para a postura adotada pelo enfermeiro no preparo da medicação figura 2, foi igual a 06. Contudo, através do estudo da imagem e considerando que a enfermeira passa nesta posição mais de um minuto durante o atendimento, realizamos o acréscimo de um ponto a este valor totalizando 07 , sendo considerada de Médio risco, necessitando de investigação mais aprofundada e breves mudanças. A figura 03 mostra esquematicamente o posto de enfermagem.

Figura 3 - Planta baixa do Posto de Enfermagem, e pontos de medição
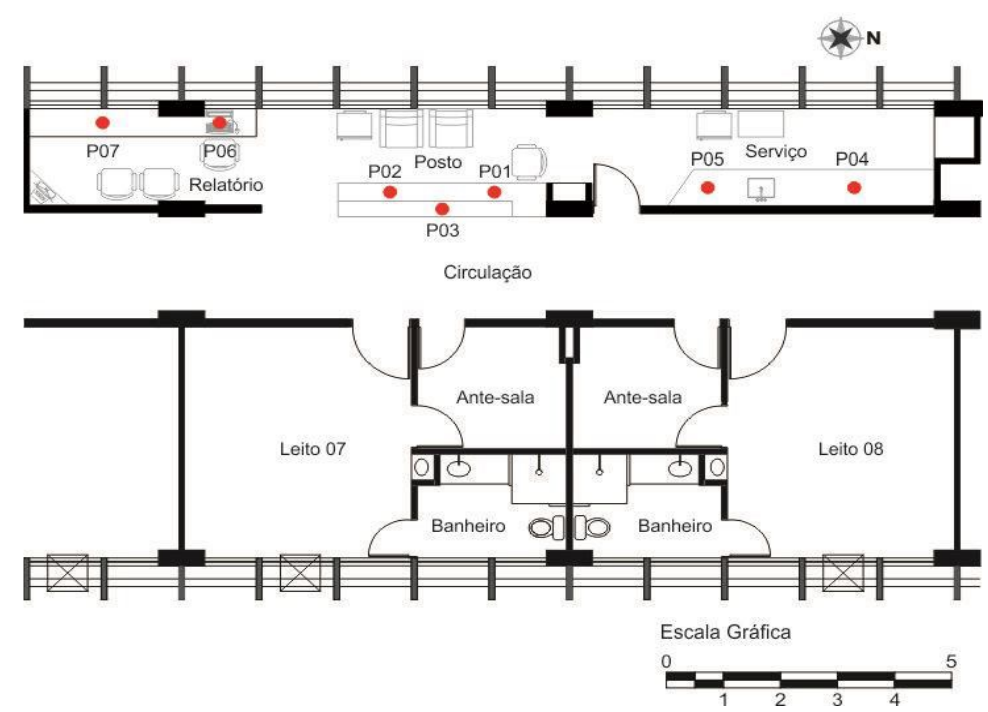

Fonte: elaboração do autor

Conforme a (ANVISA, 2002) que regulamenta o dimensionamento, a quantificação e as instalações prediais dos ambientes nos Estabelecimentos Assistenciais de Saúde, os postos de enfermagem de devem ter área mínima de $6,00 \mathrm{~m}^{2}$. O posto de trabalho utilizado como objeto de estudo possui aproximadamente $30,00 \mathrm{~m}^{2}(16,50 \times 1,85 \mathrm{~m})$. Foram elegíveis para o 
estudo 40 enfermeiros das clínicas cirúrgicas da internação do Hospital das Clínicas da UFPE. Destes, seis encontravam-se em período de férias, seis se recusaram a participar do estudo e quatro deixaram o questionário em branco. Dos 24 profissionais entrevistados todos tinham ensino superior completo e, em média 36anos de idade. O gráfico 01 mostra a distribuição dos enfermeiros quanto ao sexo. Diante dos resultados encontrados evidenciouse que $95,8 \%$ dos entrevistados são do sexo feminino. O trabalho do enfermeiro, desde a sua origem, é exercido majoritariamente por mulheres. Atualmente, de acordo com o Conselho Federal de Enfermagem (COFEN, 2011), mais de $80 \%$ da profissão é composta por mulheres, com destaque para a divisão parcelar do trabalho nesse campo de atuação. As figuras 4 e 5, mostram imagens capturadas pela câmara de infravermelho, evidenciando áreas em destaque compatíveis com síndromes dolorosas, apresentadas nos enfermeiros voluntários desta pesquisa. As regiões topograficamente evidenciadas compreendem: a região da coluna cervical, torácica, ombros e região da coluna lombar.

Figura 4 - Imagens infravermelhas expondo a região cervical, torácica e ombro direito

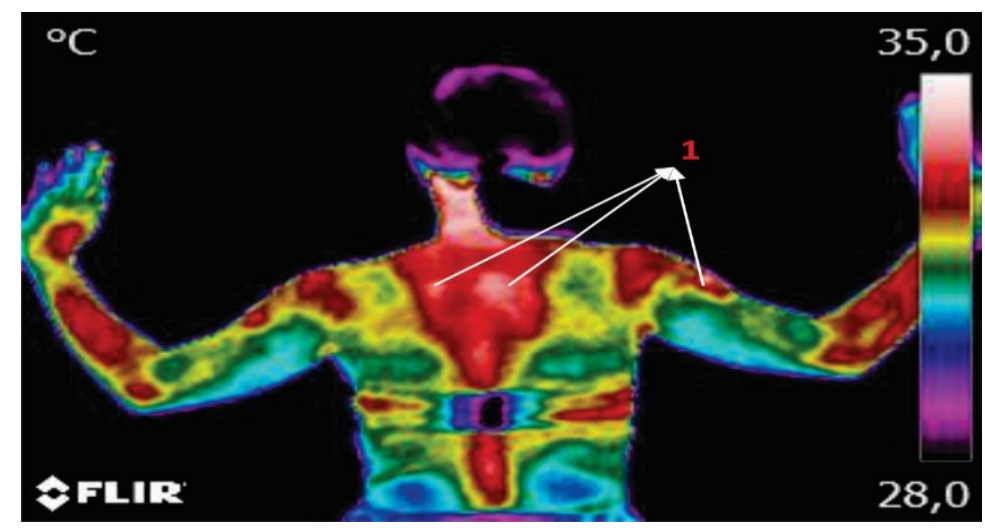

Fonte: elaboração do autor

Figura 5 - Imagens infravermelhas expondo a região cervical, coluna torácica bilateral e ombros bilaterais

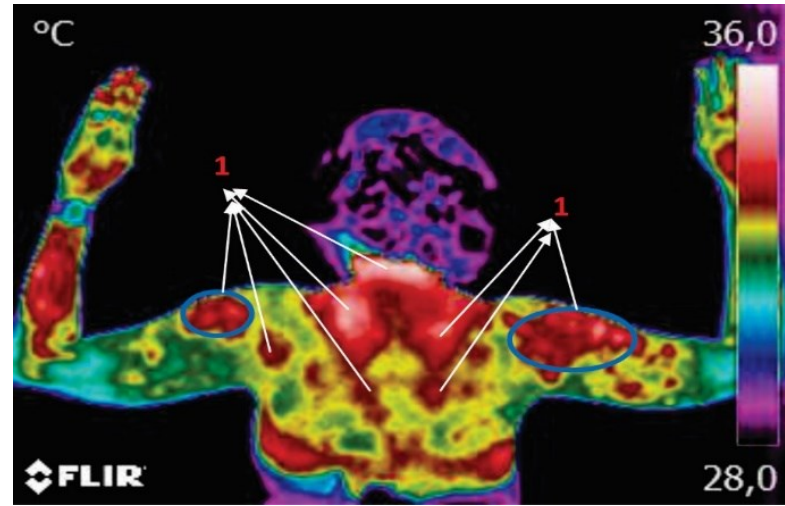

Fonte: elaboração do autor 
A topografia preponderante relacionado às principais queixas álgicas dos enfermeiros e capturada nas imagens da termografia, nesse estudo foram achadas nas regiões da coluna cervical, dos ombros e da coluna torácica e lombar.

\section{DISCUSSÃO}

Para a maioria das atividades executadas no posto de trabalho, a posição em pé é a mais adequada, para a manutenção da postura em pé, são necessários níveis baixos, porém constantes, de tensão muscular, e esse estado prolongado de contração provoca a compressão dos vasos sanguíneos, prejudicando a circulação sanguínea e resultando em transtornos como as varizes (BERENGUER, SILVA, \& CARVALHO, 2011).

A preocupação com a iluminação nos ambientes hospitalares tem se focado mais na questão econômica, envolvendo a racionalização da energia empregada e na redução dos custos de operação do sistema. Mesmo em relação aos pacientes, as discussões convergem para questões de conforto ambiental: luz de cabeceira para leitura, iluminação de vigília para permitir o acesso da equipe de enfermagem à noite sem acionar a luz de teto. Outra questão é a utilização de lâmpadas que "permitam boa reprodução de cores permitindo a identificação de alterações orgânicas", como por exemplo, a coloração da pele.

As questões de segurança são tratadas em relação à iluminação de emergência (NBR 13534-95) e (NBR 10898/99) iluminâncias médias mínimas para atividades hospitalares, considerando a dificuldade da tarefa visual, a idade do usuário, porém as legislações divergem entre os países (PECCIN, 2004). As regiões corporais admitidas topograficamente foram: as regiões da coluna cervical, dos ombros e da coluna torácica e lombar, ilustrando áreas de hiperradiação nos enfermeiros voluntários no seu local de trabalho.

Todos os voluntários relataram sentir dores nas áreas identificadas, redução da amplitude de movimento. Portanto, as possíveis disfunções identificadas e ocasionadas por diversos fatores, dentre eles o ergonômico, tem levado ao acometimento e a redução da capacidade laboral (FABRICA, 2012). Para Mafra (2010), a abordagem preventiva, é o meio ideal para lidar com estas disfunções. Vimos que nos diversos postos de trabalho do grupo avaliado, existem inúmeros riscos ergonômicos para esses profissionais, dentro de suas funções específicas, decorrente do uso excessivo da estrutura por má postura, a sobrecarga, a mecanização, a monotonia do trabalho e o esforço físico.

Retomando-se o conceito adotado no presente estudo, pode-se afirmar que as exigências do trabalho dos enfermeiros, destacando-se o ritmo e a carga de trabalho gera desgaste físico comprometendo a sua saúde (KIRCHOF, et al., 2011).

\section{CONSIDERAÇÕES FINAIS}

A ergonomia adapta as condições de trabalho as características psicofisiológicas do ser humano, procurando evitar constrangimentos e/ou lesões. Os riscos ergonômicos tornam-se cada vez mais presentes nos postos de trabalho. E para os enfermeiros das clinicas cirúrgicas do Hospital das Clínicas esses riscos caminham lado a lado influenciando no sistema de trabalho e na contribuição do processo saúde-doença devido às características próprias da atividade laboral desempenhada. No processo de avaliação, a termografia 
permitiu a correlação dos sintomas percebidos pelos enfermeiros com a identificação das áreas acometidas e sinalizadas pela radiação captadas nas imagens termográficas. Diversos estudos já demonstraram direta correlação entre a intensidade da dor e temperatura. Esta pesquisa aponta um importante problema de saúde pública e assinala uma elevada prevalência de agressões a coluna vertebral que tem acometido um percentual significante de enfermeiros. Os resultados sugerem associação de dor lombar com sexo, idade, manuseio de materiais e equipamentos e postura inadequada no trabalho. Os dados apresentados podem ser aproveitados e contribuir de maneira positiva para benefício dos enfermeiros.

\section{REFERÊNCIA}

AHN, Correlation Between Pain Scale and Infrared Thermogram in Unilateral Pain Patients After Nerve Block. (abstract). J Korean Med Thermol ., p. 59, 2002.

ABERGO - Associação Brasileira de Ergonomia. Fonte: http://ergonomiaaplicada.blogspot.com.br/2007/08/abergo-associao-brasileira-deergonomia.html. Acesso em abril de 2016.

ANVISA.Ministério da Saúde. Agência Nacional de Vigilância Sanitária. RESOLUÇÃO-RDC NN 50, DE 21 DE FEVEREIRO DE 2002. Regulamento Técnico para planejamento, programação, elaboração e avaliação de projetos físicos de estabelecimentos assistenciais de saúde. 2002.

$\mathrm{BACH}, \mathrm{V}$, et al. The interaction between sleep and thermoregulation in adults and neonates. Sleep medicine reviews, v.6, n.6, 481-492.2003.

BERENGUER, F., SILVA, D., CARVALHO, C. Influência da posição ortostática na ocorrência de sintomas e sinais clínicos de venopatias de membros inferiores em trabalhadores de uma gráfica na cidade do Recife-PE. Rev Bras Saúde Ocupacional, 153-161. 2011.

BRIOSCHI, M. Infrared thermal imaging in patients with chronic pain in upper limbs. J Korean Med Therm. 2002; 2(1):73-73., pp. 73-73. 2002.

DIEGO-MÁS, J. A., CUESTA, S. A. (NIOSH (National Institute for Occupacional Safety and Health, http://www.ergonautas.upv.es/metodos/niosh-ayuda.php. Acesso em 02 de outubro de 2015. 2015.

DOBRATZ, R. J. et al. Predicting energy expenditure in extremely obese women. J Parenter Enteral Nut, 217-27. 2007.

COFEN.http://www.cofen.gov.br/.revista.portalcofen.gov.br/index.php/enfermagem/article//19 5/131. Fonte: COFEN. Acesso em março de 2016. 2011.

CONNELL, R. W. Políticas da masculinidade. Educação e Realidade. Políticas da masculinidade. Educação e Realidade, 185-206. 2010. 
EDWIN, C. Effects of pilatesbased exercises on pain and disability in individuals with persistent nonspecific low back pain: a systematic review with meta-analysis, pp. 7080. 2011.

GOMES, M., BORGES, L. NASCIMENTO, J. Ciclos de Desenvolvimento Profissional e a Qualidade de Vida de Professores de Educação Física da Região Sudoeste da Bahia.Rev Bras Educ Física Esporte Lazer e Dança, 104-114. 2007.

INOUE, K. C. Dimensionamento de pessoal de enfermagem em Unidade de Terapia Intensiva para adultos. ACTA, 379-384. 2010.

HIGNETT, S. MCATAMNEY, L. Rapid entire body assessment (REBA). Applied Ergonomics, p201-205. 2005.

KATCH, F. I. McARDLE, W. D. (2006). Nutrição, exercício e saúde. . Rio de Janeiro: Medsi.

KIRCHHOF, A. et al. Compreendendo cargas de trabalho na pesquisa em saúde ocupacional na enfermagem. Colomb Med., 113-19. 2011.

MAFRA, S. C. A Ergonomia Como Ferramenta Para a Melhoria da Qualidade de Vida nos Departamentos e Setores da Universidade Federal de Viçosa. XII Simpep - Bauru, São Paulo: Novembro. 2010.

MORAES, A. PEQUINI, S. Ergonomia e usabilidade. Salvador: UNEB. 2004.

MORAES, A. MONT'ALVÃO, C. Ergonomia: conceitos e aplicações. Teresópolis: 4. ed. rev. atual e ampl. 2010.

MEDEIROS, S. M. As novas formas de organização do trabalho na $3^{a}$ revolução industrial e a força de trabalho em enfermagem: estudo em Natal/RN. Tese de doutorado em enfermagem/Universidade de São Paulo. Ribeirão Preto, São Paulo, Brasil: Escola de Enfermagem de ribeirão Preto. Programa de Pós-gradução Interunidades. Departamento materno infantil e saúde pública. 2006.

Ministério da Saúde. BRASIL.Ministério da Sáude.Doenças relacionadas ao trabalho. Manual de procedimentos para o serviço de saúde, Brasília (DF). 2001.

NBR 10898/99. (s.d.). Sistema de Iluminaçao de Emergencia.

NBR 13534-95. (s.d.). NBR- NORMAS PARA INSTALAÇÕES ELÉTRICAS .

NBR10152. TÉCNICAS, ASSOCIAÇÃO BRASILEIRA DE NORMAS. Nível de rúido para conforto acústico. 2000.

NBR5413. Iluminação de interiores. 1992.

NBR8995-1. NBR ISSO/CIE 8995-1. Iluminação de ambientes de trabalho. 2013.

PANERO, Julius, MARTINS Zelnik. Dimensionamento humano para espaços interiores. Barcelona: Editora Gustavo Gili,7ª edição, 2012.

PASCHOAL, T.TAMAYO, Á. Validação da Escala de Estresse no Trabalho. Estudos de Psicologia. Universidade de Brasília. , 45-52. 2004. 
PINHEIRO, F. A. TRÓCOLI, B. T. CARVALHO, C. V. Validação do Questionário Nórdico de Sintomas Osteomusculares como medida de morbidade, Rev Saúde Pública, 307312. 2005.

POLIT, D. BECK, B. HUNGLER. Fundamentos de pesquisa em enfermagem: métodos, avaliação e utilização. Porto Alegre: Artmed. 2004.

RIBEIRO, S. O. O processo de trabalho em enfermagem. pp. 616-623. 2003.

SOARES, M. M. Fundamentos em Ergonomia. . Apostila do primeiro Curso de Mestrado em Ergonomia. Departamento de Design. Recife, Pernambuco: Universidade Federal de Pernambuco. 2013.

STACCIARINI, J.TRÓCOLLI, B. Instrumento para mensurar o estresse ocupacional: inventário de estresse Rev.latino-am.enfermagem. Ribeirão Preto, v. 8, n. 6, 40-49. 2000.

UNBEHAUM, S. G. Gênero, masculinidade e prevenção. . Anais do seminário sobre cultura, saúde e doença. Londrina: Universidade Estadual de Londrina., 150-162. 2013.

VIVIANI, M. T. e colaboradores. Correlação entre o gasto energético, peso corporal e massa magra de mulheres sedentárias e ativas. Colloquium Vitae. São Paulo. Vol. 1. Núm. 2., 75-83. 2009.

VOLP, A. C. e colaboradores. Energy expenditure: components and evaluation methods. Nutr Hosp. Vol. 26. Núm. 3. Nutr Hosp. Vol. 26. Núm. 3. , 430- 440. 2011. 